Mots. Les langages du politique

$86 \mid 2008$

Toponymes. Instruments et enjeux

\title{
Collectif, Démocratie participative en Europe
}

Thomas Frinault

\section{(2) OpenEdition}

Journals

Édition électronique

URL : https://journals.openedition.org/mots/13822

DOI : $10.4000 /$ mots. 13822

ISSN : 1960-6001

\section{Éditeur}

ENS Éditions

\section{Édition imprimée}

Date de publication : 30 mars 2008

Pagination : 119-122

ISBN : 978-2-84788-129-5

ISSN : 0243-6450

\section{Référence électronique}

Thomas Frinault, «Collectif, Démocratie participative en Europe », Mots. Les langages du politique [En ligne], 86 | 2008, mis en ligne le 30 mars 2010, consulté le 24 avril 2022. URL : http:// journals.openedition.org/mots/13822 ; DOI : https://doi.org/10.4000/mots.13822 


\title{
Comptes rendus de lecture
}

\author{
Démocratie participative en Europe \\ Collectif \\ Sciences de la société, nº 69 (mai 2006)
}

Alors que le paradigme participatif s'impose peu à peu dans les espaces scientifiques, médiatiques et politiques, ce numéro 69 de la revue Sciences de la société se propose d'étudier le thème de la « démocratie participative en Europe» à travers un dossier composé d'une douzaine d'articles issus d'horizons disciplinaires variés. Comme le rappelle Stefan Bratosin dans l'article introductif du dossier, le succès de cette notion risque de l'emporter sur sa précision conceptuelle. Concluant à la difficulté théorique de saisir les sens pluriels de la notion de démocratie participative, le dossier se fait l'écho de cette complexité en privilégiant un questionnement relatif aux pratiques. Mais ce parti pris (ou choix contraint?) n'est pas sans effet sur la nature kaléidoscopique de l'ensemble et sur l'absence de clarification conceptuelle.

La progression des articles n'étant pas évidente à saisir, il est possible de réunir les trois contributions abordant l'aspect communautaire et justifiant de ce fait la dimension européenne du dossier. L'article de Janie Pélabay revient sur le projet de l'UE d'œuvrer par l'éducation à l'avènement d'une démocratie européenne en favorisant l'association des citoyens au processus d'intégration européenne et en veillant à l'exigence d'unité dans une Europe de plus en plus large et donc plurielle. Tel est par exemple l'objectif du programme "Citoyens pour l'Europe», qui privilégie l'expérience vécue de la démocratie européenne en misant sur le développement des «bonnes pratiques» démocratiques. Cette démarche donne une résonance directe aux critiques qualifiées de (néo-)républicaines vis-à-vis du courant libéral et multiculturaliste. Le courant néo-républicain, soucieux de mettre simultanément l'accent sur l'unité et les devoirs des citoyens, met ainsi en garde contre la fragmentation des sociétés démocratiques liée à la pluralité des valeurs et des cultures. Mais pour l'auteur, le risque existe que la participation citoyenne ne s'efface rapidement devant la promotion par le haut d'un «bien commun » européen étouffant les visions individuelles et la diversité des modes de vie prônées par le libéralisme politique. À l'évidence, une connaissance préalable de la philosophie politique peut ici être utile à une meilleure compréhension de 
l'article et de ses enjeux. L'article de François Foret étudie la dimension religieuse du projet européen de démocratie participative visant à relégitimer la construction européenne par une densification du dialogue avec la société civile. Les acteurs religieux y apparaissent comme des interlocuteurs privilégiés. L'auteur explore, avec un talent d'écriture indéniable, les relations des Églises à l'engagement politique, notamment européen, et aux médias. En revanche, la dimension communautaire de l'article semble rapidement céder le pas devant sa dimension européenne. L'article de Muriel Rambour, portant sur l'association de la société civile aux travaux conventionnels de rédaction d'une Constitution européenne, montre de manière très claire comment la consultation, qui a été menée auprès de membres de la société civile organisée (ONG, associations professionnelles, organisations de base...) et non de citoyens ordinaires, pose une série de questions : aspect catégoriel et sectoriel des revendications, représentativité (ce sont des membres de la société civile et non des représentants), caractère expéditif de la collecte des avis. Cette consultation, tout en renforçant la légitimité de la procédure, laisse finalement de côté les citoyens qui ne participent pas à une structure ayant plus ou moins directement l'Europe comme centre d'intérêt.

La seconde série d'articles, soit un total de huit, justifie plus difficilement la dimension européenne revendiquée par le dossier. Ce qui pose la question non résolue: pourquoi insister a priori sur l'ancrage européen du dossier pour en dire aussi peu par la suite? L'article de Jean-Louis Beauvois passe en revue les tensions contradictoires inhérentes à l'exigence de démocratie participative en ouvrant des pistes de réflexion intéressantes. Mais l'analyse reste trop en surface. Tout comme celle de Maurice Blanc qui applique le concept sociologique de transaction sociale à la démocratie participative. L'article semble hésiter entre une vraie théorisation ou une démonstration par la preuve. Celui de Laurence Monnoyer-Smith esquisse les contours d'une nouvelle construction théorique de la délibération fondée sur la théorie de l'agir créatif de Hans Joas et opposable à la théorie habermassienne de l'agir communicationnel. Après une critique d'un modèle délibératif idéal, trop exclusivement polarisé sur le seul échange langagier et argumentatif, l'auteur plaide pour une prise en compte de la créativité dans la procédure délibérative elle-même. Mais les nouveaux supports de créativité qu'elle mentionne ne sont jamais réellement abordés. En outre, le lien avec la démocratie participative parait, au minimum, lâche et indirect. Envisageant les rapports entretenus par la démocratie participative avec les différents échelons territoriaux, l'article de Sandra Breux montre comment cette forme de démocratie met en jeu un principe de territorialité différent de celui de la démocratie représentative: un territoire choisi et non pas imposé par une entité politique supérieure. Mais l'auteur semble plus s'intéresser à la question du lien entre citoyenneté, communauté et territoire qu'à l'articulation entre démocraties représentative et participa- 
tive. Après avoir rappelé la désaffection des jeunes Suédois pour la politique, Peter Dahlgren et Tobias Olsson montrent comment Internet peut faciliter l'engagement politique et faire évoluer les différentes dimensions des cultures civiques: connaissances, valeurs, confiance, espaces de communication, pratiques, identités. À partir de la restitution d'entretiens, ce qui confère une dimension plus incarnée au propos, les auteurs donnent à voir une implication citoyenne facilitée par Internet plus que réellement transformée. L'article de Constantin Salavastru prend prétexte de la démocratie participative pour étudier de manière savante, et parfois difficile d'accès, la question du langage, de l'argumentation, des moyens verbaux et des procédures stylistiques. L'idée la plus intéressante réside dans le paradoxe de la démocratie participative censée ouvrir le pouvoir mais obéissant à des règles rhétoriques complexes et inaccessibles à une majorité de citoyens ordinaires. Enfin, deux articles, étiquetés «Arguments», referment ce dossier. Celui d’Éric George, qui s'interroge sur les liens entre État, marché et société et plus spécifiquement sur les formes de mobilisation sociale : selon l'auteur, elles illustreraient la possibilité d'une démocratie continue reposant sur un contrôle permanent des représentés sur les représentants. Et celui de Nikos Smyrnaios, qui soulève le problème de la confusion entre les usages potentiels d'Internet, attachés à une vision originelle de l'internaute idéal, et les usages effectifs soumis à des facteurs exogènes. Deux articles de réflexion intéressants mais qui développent un propos très généraliste.

Finalement, ce que le lecteur peut redouter à la lecture de l'introduction se confirme par la suite: l'absence de définition unifiée de ce qu'est la démocratie participative conduit à multiplier les angles d'analyse. Sandra Breux la définit comme l'ensemble des actes par lesquels les citoyens interviennent dans le processus de décision publique et influencent les décisions des gouvernants. Or cette association active au processus décisionnel ne transparait que trop rarement dans les articles. La différence, pourtant essentielle, entre un dispositif de participation institutionnalisé par le haut et des logiques de participation provenant du bas n'est jamais posée. Pour beaucoup de développements, l'entrée pertinente semble finalement être ailleurs: espace public, militantisme, lobbying, citoyenneté. La partition entre société civile organisée et citoyens ordinaires, évoquée à juste titre par Muriel Rambour, aurait mérité d'être mieux considérée. En définitive, le sentiment est celui d'une collection hétéroclite d'articles permise par le flou conceptuel et qui n'aide pas à saisir ce qu'il y a d'irréductible dans l'idée de démocratie participative. L'analyse aurait été plus convaincante à moins multiplier les illustrations ponctuelles et à davantage mobiliser des récits détaillés ou études de cas sur tel ou tel dispositif. Mais le format souvent court des articles, induit par leur nombre élevé, a imposé il est vrai de fortes contraintes aux contributeurs de ce numéro. Ces derniers ont pu par ailleurs manquer d'espace pour 
expliquer au lecteur profane les enjeux scientifiques des sujets abordés. Un impératif d'autant plus fort pourtant que, le dossier étant pluridisciplinaire, il ne s'adressait a priori pas à un public de spécialistes.

Thomas Frinault

CRAPE, IEP de Rennes

Les trois écritures. Langue, nombre, code

Clarisse Herrenschmidt

2007, Paris, Gallimard, $510 \mathrm{p}$.

"Les mots nous disent ce que nous, en tant que société, nous croyons qu'est le monde», écrit Alberto Manguel dans son livre consacré aux mots et au monde (2000, p. 15). Jorge Luis Borges ne pensait-il pas que l'univers pouvait se confondre avec une vaste bibliothèque (1995, p. 111) et que la clef se situerait peut-être dans les caractères de l'alphabet?

Clarisse Herrenschmidt, linguiste et anthropologue, nous invite à revisiter l'écriture des langues ${ }^{1}$, le langage des nombres (en tant qu'entité arithmétique) et l'encodage informatique, non pas du point de vue du sens, mais selon un certain rapport entre la façon d'écrire et la façon de penser: «Nous cherchons les structures: comment certaines écritures notant les langues instaurent, expriment et expliquent le rapport des choses du monde et des choses du langage tel que l'ont vécu les vivants qui s'en servaient.» (p. 18-19) Un voyage dans le temps et dans l'espace dont elle déplore les limites, puisque les territoires visités se limitent à la Mésopotamie, l'Iran, la Méditerranée orientale et le monde grec, l'Europe et les états-Unis pour la période récente. Le chinois, vraisemblablement la seule écriture non alphabétique encore en vie (Marchand, 2002, p. 14), n'est donc pas étudié, mais cela est consistant avec l'approche revendiquée par l'auteur de se limiter à l' "Occident».

Des civilisations littéraires telles que la Perse, la Grèce, l'Italie et la civilisation juive, nées à partir du deuxième millénaire avant notre ère, sont toujours vivantes. La naissance des fondements graphiques du Proche et MoyenOrient, de l'Europe et de l'Occident modernes - sans oublier l'Inde ou la Chine - relève de l'histoire très ancienne, mais «cette Antiquité, qui paraît si lointaine, ne l'est point au regard des écritures» (p. 102) et cela rend encore plus passionnant le voyage.

L'histoire inventive des langues, qui constitue la première partie de l'ouvrage, débute vers 3300 avant notre ère et s'achève au plus tard vers 750 avant notre ère avec l'alphabet grec complet.

1. Et l'apprentissage de l'écriture et de la lecture, dans une critique acerbe de l'enseignement "global», qui figure dans les p. 62-63 de l'ouvrage. 